\title{
DISPONIBILIDAD DE RECURSOS LÍTICOS Y EXPLOTACIÓN HUMANA DE LA DIVISORIA ANDINA. VALLE DEL RÍO DE LAS TAGUAS, SAN JUAN, ARGENTINA
}

\author{
AVAILABILITY OF LITHIC RESOURCES AND HUMAN EXPLOITATION OF THE \\ ANDEANBORDERLANDS. RIO DE LAS TAGUAS VALLEY, SAN JUAN, ARGENTINA
}

\author{
Valeria Cortegoso $^{1}$, Víctor Durán ${ }^{1}$, Silvina Castro $^{2}$ y Diego Winocur ${ }^{3}$
}

\begin{abstract}
A través del análisis de disponibilidad y explotación de recursos líticos, se exploran características de la ocupación del límite argentino-chileno. El área se localiza en el NO de San Juan (Argentina) y comprende desde el límite de vertientes cordilleranas $(4.500 \mathrm{msm})$ hasta los valles próximos $(3.700 \mathrm{msm})$. Se definen patrones espaciales para el uso de estos ambientes considerando: variaciones altitudinales, caracterización de fuentes de materias primas líticas y actividades de talla asociadas. Los sectores más altos, con fuentes primarias, muestran estadios iniciales de la secuencia reductiva y menor densidad de elementos líticos. Los sectores más bajos muestran altas densidades y secuencias completas asociadas a fuentes secundarias. Se elaboran propuestas preliminares sobre los patrones temporales de ocupación considerando la composición del registro lítico en un sitio con una secuencia de ca. 9.000 años y los datos paleoambientales disponibles. Según esta aproximación, el área habría sido explorada luego de la última retirada de los hielos. Las ocupaciones humanas sucesivas coinciden temporalmente con períodos de disponibilidad hídrica en cordillera y aridez en altitudes menores de la vertiente occidental. Teniendo en cuenta las características topográficas, el registro arqueológico y los datos paleoambientales, se propone que el área habría sido explotada desde esa vertiente.
\end{abstract}

Palabras claves: disponibilidad de recursos líticos, explotación humana, divisoria andina, San Juan, Argentina.

Through an analysis of the availability and exploitation of lithic resources, characteristics of human occupations in the ArgentineanChilean boundary are discussed. The area is located in NW San Juan (Argentina) and extends from the edge of the mountain range slopes $(4,500 \mathrm{masl})$ to the nearby valleys $(3,700 \mathrm{masl})$. We define spatial patterns for the use of these environments considering: altitudinal variation, characterization of lithic raw material sources, and associated knapping activities. The higher areas, with primary sources, evidence the first lithic reduction sequence stages and a lower density of elements. The lower sections show high densities and complete sequences associated with secondary sources. We elaborate a preliminary record of occupation, through a consideration of the lithic record at a site with a ca. 9,000-year sequence and available paleoenvironmental data. According to this approach, the area would have been explored after the last retreat of the ice. Successive human occupations coincide with periods of water availability in the high cordillera and aridity at lower altitudes on the western slopes. Given the topography of the area, the archaeological record, and paleoenvironmental data, is proposed that the area would have been exploited from bases on the mountain western flanks.

Key words: Lithic resources availability, human exploitation, Andean borderlands, San Juan, Argentina.

Este trabajo explora a través del análisis de disponibilidad y explotación de recursos líticos las características de la ocupación humana del valle del río de Las Taguas a lo largo del Holoceno. El área se encuentra ubicada en el NO de la provincia de San Juan (Argentina). Comprende en parte el límite de vertientes cordilleranas entre las latitudes $29^{\circ} 10^{\prime} y$ $29^{\circ} 30^{\prime} \mathrm{S}$ y los valles inmediatos en el sector oriental que corresponden a la ladera oeste del extremo sur de la Cordillera de la Ortiga. Las altitudes varían entre $4.500 \mathrm{msm}$ en el límite argentino-chileno, hasta $3.700 \mathrm{msm}$ en el fondo de los valles. Hacia el este, el territorio de la provincia de San Juan tiene otros cordones montañosos, como el de Colangüil, con altitudes aún mayores que el límite internacional, alcanzando su divisoria los $5.000 \mathrm{msm}$. Por el contrario, la vertiente occidental de los Andes tiene vías muy accesibles a valles importantes, a través de los arroyos que forman las nacientes del río Huasco (Figura 1).

El conocimiento del uso humano de este ambiente puede ser entonces de importancia para

1 Consejo Nacional de Investigaciones Científicas y Técnicas, Universidad Nacional de Cuyo, Mendoza, Argentina. Facultad de Filosofía y Letras. Parque Gral. San Martín s/n. vcortegoso@gmail.com; duranvic@logos.uncu.edu.ar

2 Agencia Nacional de Promoción Científica y Tecnológica. Universidad Nacional de Cuyo, Mendoza, Argentina. silcastro24@gmail.com

3 Universidad de Buenos Aires, Buenos Aires, Argentina. diegowinocur@hotmail.com 


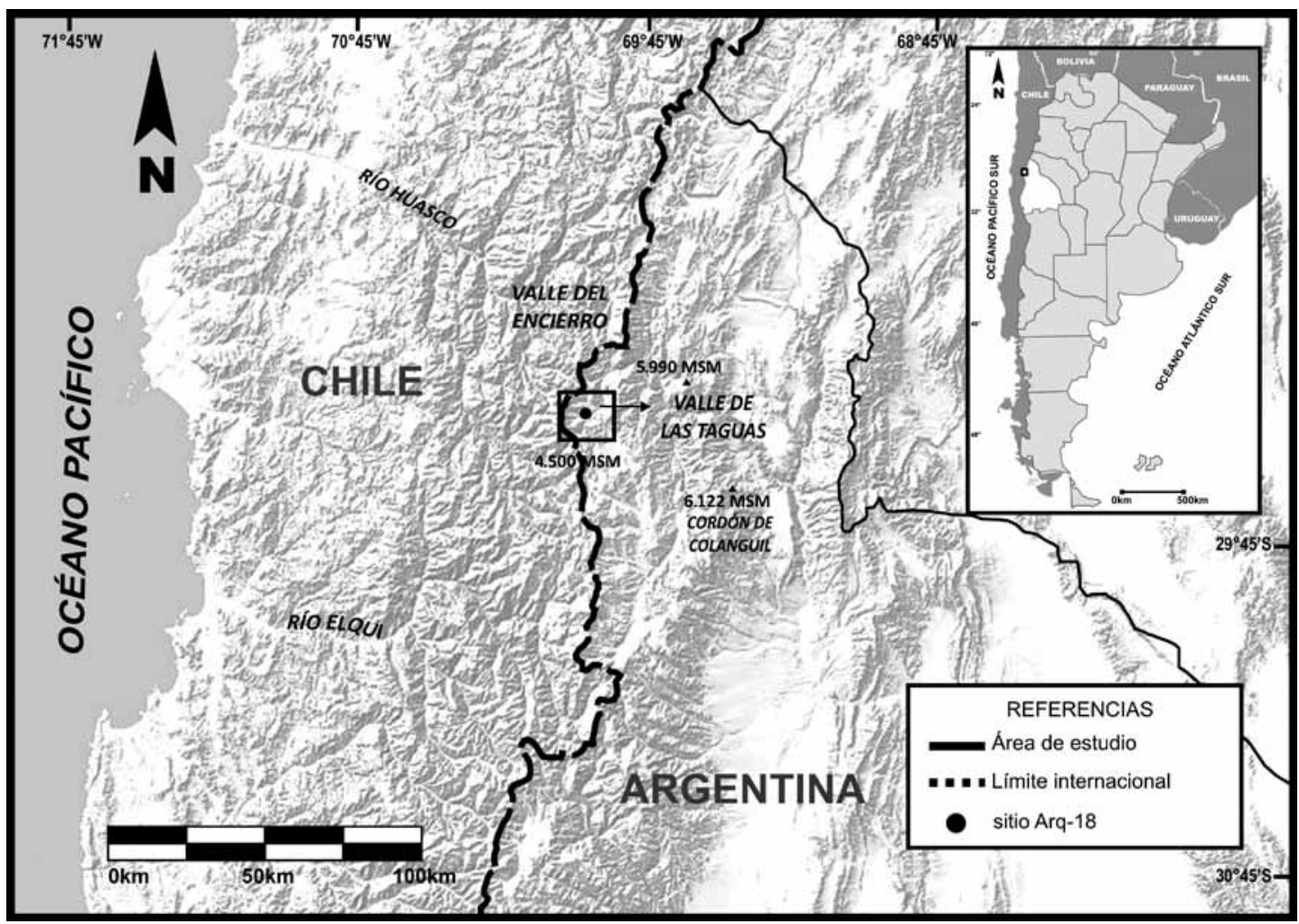

Figura 1. Localización del área. Se destaca la altitud de los principales cordones montañosos y los valles en el sector occidental y oriental de la cordillera de los Andes.

Study area. Map shows the altitude of the main mountain ranges and valleys of the western and eastern Andean slopes.

comprender la historia de poblaciones localizadas a uno y otro lado de la cordillera. Se trata de un área con una disponibilidad rica en recursos bióticos y abióticos; durante las temporadas estivales una gran cantidad de arroyos y vegas ofrecen sustento a grandes manadas de camélidos (Lama guanicoe) que aún hoy son visibles en el paisaje. Según se analizará en este trabajo, el área también tiene una importante oferta de recursos líticos, que habrían sido explotados de forma diferencial a lo largo de la historia de la ocupación humana de este ambiente.

Entre 2004 y 2007 se realizaron prospecciones sistemáticas que permitieron relevar casi treinta sitios arqueológicos en el área. Muchos de estos sitios son a cielo abierto y con estructuras pircadas, pero se detectaron también refugios naturales acondicionados. Se realizaron recolecciones sistemáticas de superficie y excavaciones que han dado como resultado la existencia de un registro de material lítico integrado por más de 30.000 piezas. Según las excavaciones en aleros, que han mostrado la mayor profundidad temporal, los primeros vestigios de explotación del área se remontan a comienzos del Holoceno. Los resultados se apoyan en una secuencia cronológica obtenida en un sitio estratificado (ARQ-18) para el cual se han obtenido 18 fechados radiocarbónicos convencionales y AMS, comprendidos entre 9.000 y 1.500 años a.p.

Este trabajo se centrará en el análisis de las fuentes primarias y secundarias de materias primas líticas que se relevaron en el área, poniendo énfasis en la explotación diferencial de acuerdo a la variación altitudinal de las mismas. Las fuentes se localizan en sectores que van desde la divisoria cordillerana hasta los valles y ofrecen materias primas de rocas silíceas criptocristalinas con calidades variables para la talla. Las particularidades de las materias primas en los distintos sectores y las condiciones generales de los ambientes según su altitud son fundamentales para comprender cómo fueron explotados estos recursos y cuáles son las actividades humanas asociadas a cada una de las fuentes.

Se presentará la caracterización y disponibilidad de los recursos líticos en el área, y una propuesta 
preliminar sobre la variabilidad espacial y temporal en la explotación de los mismos. Para ello se analizan algunos aspectos diacrónicos del uso del área teniendo en cuenta la composición del registro arqueológico y los datos paleoambientales disponibles para la región inmediata y áreas vecinas. Se intenta de este modo aportar al conocimiento de la utilización de ambientes de altura a lo largo del Holoceno como un modo de mejorar las bases para comprender el poblamiento humano de la macro región y la vinculación entre sociedades de ambas vertientes cordilleranas.

\section{Aspectos Metodológicos}

Desde un análisis basado en la organización tecnológica (sensu Nelson 1991) los estudios buscan evaluar la variabilidad temporal en los patrones de explotación de recursos líticos en la región. Los principales cambios en las estrategias tecnológicas que han comenzado a estudiarse intentarán ser referidos a sistemas de abastecimiento y diseño de instrumentos que caracterizan las sociedades de ambas vertientes andinas a lo largo del Holoceno.

En los análisis se procura determinar no sólo el tipo y la calidad de la materia prima explotada y/o transportada, sino también el estado en que se traslada y los episodios del proceso reductivo que pueden reflejarse en cada uno de los sitios (Andrefsky 2009; Steffen et al. 1998; entre otros). A lo largo de las sucesivas ocupaciones del área se busca detectar cambios en el modo en que las materias primas de las distintas fuentes fueron tratadas; esto incluye el tipo de soportes trasladados, patrones de consumo y aprovisionamiento tecnológico (sensu Kuhn 2004).

La metodología utilizada para la detección de estas fuentes fue la prospección directa sistemática de la región en cada uno de sus ambientes. Las fuentes primarias y secundarias relevadas han sido agrupadas según su localización en tres grandes áreas con diferencias altitudinales importantes: divisoria cordillerana (4.500-4.200 msm), sectores intermedios (Arroyo Potrerillos 4.000-3.800 msm) y fondo de valles (Las Taguas y Los Amarillos, por debajo de $3.800 \mathrm{msm}$ ). Se busca a través del análisis comparativo de los núcleos, productos de talla y artefactos formatizados en cada uno de los sectores, establecer diferencias en cuanto a la explotación de materias primas en los distintos ambientes. En el caso de las fuentes secundarias localizadas en el fondo de los valles, donde los productos de talla son mayoritarios, también se han realizado comparaciones de grano más fino de las secuencias de reducción a partir del análisis de los atributos morfotecnológicos (Aschero 1983; Cortegoso 2008). Se busca de este modo comprender la posible variabilidad en los procesos de talla y en consecuencia la funcionalidad de los sitios dentro de estos ambientes más favorables para la instalación humana.

Para la clasificación de las materias primas se utiliza una tipología basada en la descripción litológica macroscópica que incluye tipos generales (silíceas, cuarzos, etc.) y subtipos en base a cualidades como: color, textura, brillo y translucidez. Como complemento de esta clasificación macroscópica se realizan análisis petrográficos sobre cortes de lámina delgada para obtener una caracterización precisa de los recursos líticos predominantes. Esta metodología ha sido aplicada en el Centro Oeste Argentino (COA) para comprender la variabilidad en el uso de recursos líticos en el Holoceno medio y tardío con resultados interesantes para la explicación de cambios en la subsistencia y la movilidad (Cortegoso 2008).

\section{Localización de las Fuentes}

Casi todos los recursos líticos que se han detectado hasta ahora corresponden a rocas silíceas criptocristalinas de distinta calidad y coloración. Según se mencionó, las fuentes se ubican en tres grandes áreas con particularidades en cuanto a la altitud, topografía y condiciones ambientales. Se efectuará una caracterización geológica de las fuentes y del registro arqueológico asociado a cada una de ellas, comenzando por los sectores más altos, luego se señalarán las particularidades de los relevamientos, la composición de las muestras $\mathrm{y}$, por último, se hará una comparación entre las fuentes ubicadas a mayor altitud y las localizadas en los valles.

\section{Divisoria y sectores intermedios}

El sector más alto donde se han localizado fuentes primarias abarca el límite argentino-chileno y una zona próxima que comprende un poco más de un kilómetro hacia el oriente (4.500-4.200 msm). Se trata de una peneplanicie extensa sin cobertura vegetal y con una leve inclinación regional hacia el este (Figura 2). La superficie presenta un alto grado 


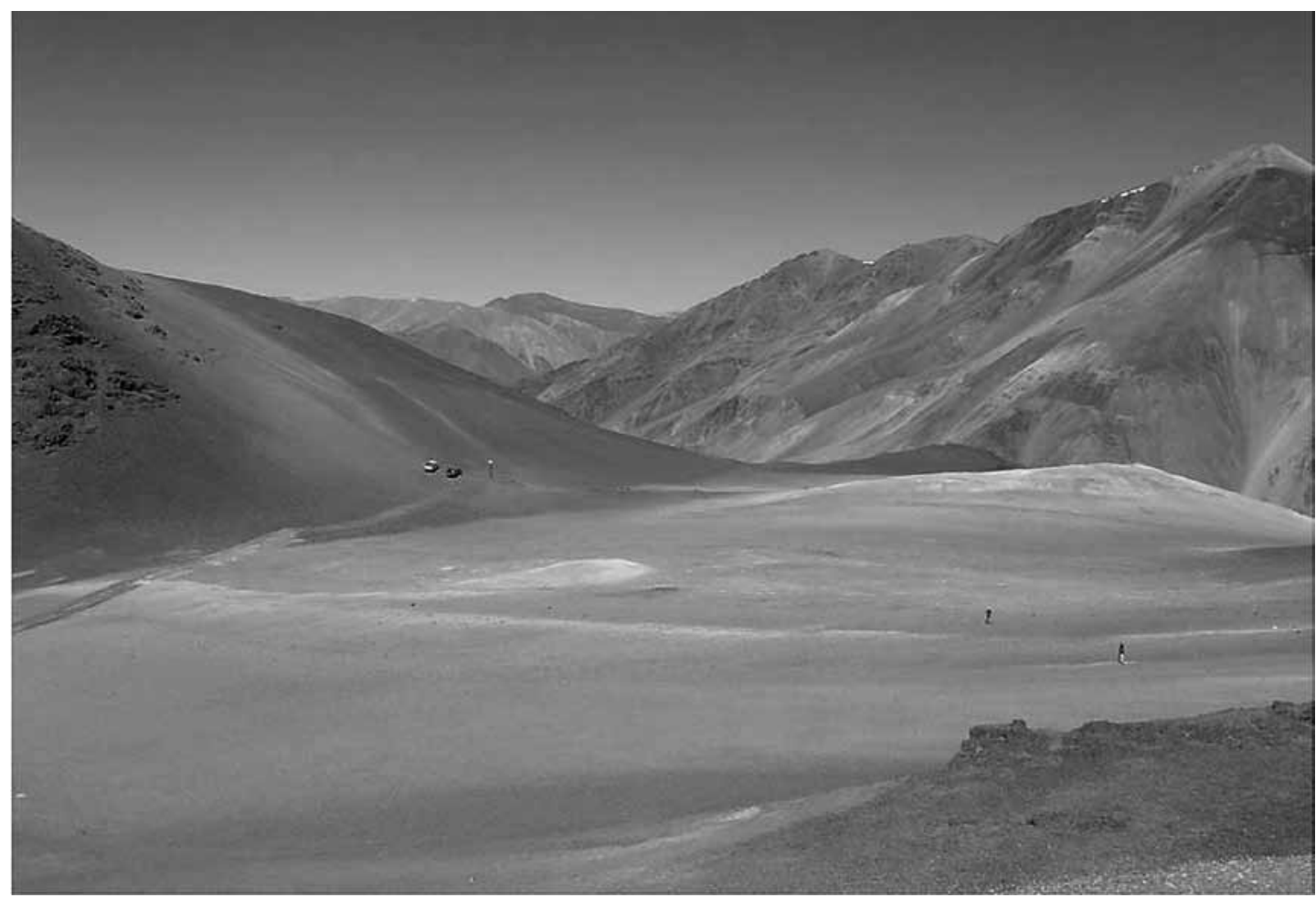

Figura 2. Divisoria cordillerana argentino-chilena. Hacia el fondo, el hito señala el límite internacional. Se observa la topografía, relieve y características del ambiente.

Argentine-Chilean Andean Frontier. In the background there is a monument marking the international boundary. Shows the topography, relief, and environmental characteristics.

de erosión, ya que la zona es afectada por procesos criogénicos. Se observan geoformas típicas de ambientes periglaciares, como guirnaldas de piedra y suelos poligonales. Esta zona presenta una amplitud térmica muy variada, tanto diaria como estacional. Este efecto térmico asociado a la escasa presencia de afloramientos y zonas de reparo genera una gran superficie donde los vientos son constantes y en situaciones extraordinarias superan los $100 \mathrm{~km} / \mathrm{h}$. Debido a estas características la meteorización física de las rocas es muy intensa y se puede observar en el patrón de fracturamiento extremo en los afloramientos circundantes y el grado a degradación del paisaje en la zona peneplanizada. Por otra parte, los procesos abrasivos generados por la acción eólica cubren con una pátina denominada pavimento del desierto la gran mayoría de estas superficies de altura.

Se localizaron allí algunos nódulos/núcleos y productos de talla sobre silíceas criptocristalinas muy dispersos. Las rocas presentan un importante grado de fragmentación natural por los procesos térmicos descritos que afectan la calidad del recurso y dificultan la visibilidad arqueológica de las actividades de talla. En las piezas más grandes es prácticamente imposible distinguir con certeza los guijarros de fractura natural, de los núcleos sumarios con planos de fractura que siguen los clivajes de la roca.

Los afloramientos de las rocas silíceas criptocristalinas mencionadas se encuentran en las laderas de los cerros circundantes y muestran una variedad de colores rojos, verdes y amarillentos. El área relevada cubre una superficie de $22.938 \mathrm{~m}^{2}$. En nueve puntos que cubren un área de $1.146 \mathrm{~m}^{2}$ se recolectaron: 71 nódulos/núcleos, dos productos de talla y un artefacto formatizado con retoques sumarios, lo que representa una densidad de 0,06 elementos por $\mathrm{m}^{2}$ (Tabla 1 ).

A un kilómetro del límite se localizó el otro afloramiento también asociado a la presencia de núcleos y productos de talla, en este caso las silíceas muestran una variedad importante de colores (rojo/mostaza, gris/celeste, crema/amarillento, crema/verdosas). Este sector es más restringido 
y presentaba mayor concentración de elementos arqueológicos, lo cual permitió establecer unidades de recolección más sistemáticas. La mayor presencia de materiales se manifestó cerca de los afloramientos. En las pendientes inmediatas los materiales se dispersaban cubriendo una superficie amplia e irregular de alrededor de $16.000 \mathrm{~m}^{2}$. Se efectuaron allí 14 unidades circulares de recolección de $5 \mathrm{~m}$ de radio cada una $\left(78,5 \mathrm{~m}^{2}\right)$. De esta forma se cubrió un área de $1.099 \mathrm{~m}^{2}$ de superficie, lo que representa el 6,9\% de la superficie de la fuente. La muestra obtenida en esta recolección está compuesta por: 82 núcleos, 250 productos de talla y tres artefactos. La densidad de elementos en la superficie de este sector es de 0,3 elementos por $\mathrm{m}^{2}$ (Tabla 1 ).

El segundo sector donde se han detectado fuentes primarias está a menor altitud y comprende espacios con acarreos sobre las laderas próximas a arroyos cordilleranos. En uno de los taludes de escombros de la margen derecha del arroyo Potrerillos (3.800 msm) se relevaron 11 nódulos y núcleos de silíceas criptocristalinas de color blanco/ crema veteado con tonos grises, de grano fino, no translúcida y opaca.

En la margen izquierda del mismo arroyo se localizaron concentraciones de núcleos y productos sobre dos niveles de terraza, algunos de estos elementos de talla corresponden a la fuente de la margen opuesta, pero son más abundantes las silíce de colores rojos con inclusiones blancas cuyos afloramientos primarios están más alejados. Los mismos se ubicarían en el sector medio del arroyo del Guanaco Zonzo, en el valle subsiguiente en dirección sur. Se trata de una roca volcánica de composición dacítica a riolítica, de coloración rojiza la cual presenta fenocristales de cuarzo inmersos en una matriz de vidrio volcánico. Estas rocas se presentan aflorando en la ladera sur del arroyo del Guanaco Zonzo inmediatamente por encima de la terraza aluvial del mismo. Se encuentran allí depositadas debido a un deslizamiento con su zona de arranque ubicada a $300 \mathrm{~m}$ por encima del depósito. Esto genera que la roca no esté en su posición original y a su vez se encuentre fracturada $\mathrm{y}$ en forma de grandes bloques.

El rescate de materiales en las terrazas del arroyo Potrerillos se realizó a partir de 29 unidades de recolección de $5 \mathrm{~m}$ de radio. En la terraza alta, que tiene una superficie de $1.800 \mathrm{~m}^{2}$, se realizaron cuatro unidades, cubriendo así $314 \mathrm{~m}^{2}$ lo que representa un relevamiento del $17,4 \%$ del sitio. $\mathrm{La}$ muestra recuperada está integrada por: 10 núcleos, 93 productos de talla y un artefacto formatizado. La densidad de materiales en este sector es de: 0,3 elementos por $\mathrm{m}^{2}$. La terraza baja es más extensa, tiene un área de $28.500 \mathrm{~m}^{2}$, allí se realizaron 25 unidades de recolección, cubriendo $1.962,5 \mathrm{~m}^{2}$. Esto representa un relevamiento del 6,88\% del sitio, que tiene una densidad de 0,6 elementos por $\mathrm{m}^{2}$. La muestra está compuesta por: 34 núcleos, 1.184 productos y cinco artefactos formatizados (Tabla 1).

En la Figura 3 se establece una comparación entre las muestras recolectadas en los sectores altos e intermedios, desde la divisoria hasta la terraza más baja del arroyo Potrerillos. La figura muestra claramente cómo a medida que disminuye la altitud de los emplazamientos, disminuye la presencia de nódulos/núcleos e inversamente, aumenta la proporción de los productos y por tanto de las actividades de talla en los distintos sitios. Esto tiene relación tanto con la disponibilidad y calidad de los recursos líticos en estos emplazamientos, como con las condiciones ambientales en cada uno de ellos. El sector más alto de la divisoria muestra una muy

Tabla 1. Composición de las muestras líticas y densidad de elementos por $\mathrm{m}^{2}$ en las tres áreas. Composition of the lithic samples and density of elements per $m^{2}$ in the three areas.

\begin{tabular}{llcccc}
\hline \multicolumn{1}{c}{ Fuentes y altitud } & Sectores & $\begin{array}{c}\text { Nódulos/ } \\
\text { núcleos }\end{array}$ & Productos & Formatizados & Densidad \\
\hline $\begin{array}{llccc}\text { Divisoria Andina } \\
4.500-4.200 \mathrm{msm}\end{array}$ & Sector Alto & 71 & 2 & 1 & 0,06 \\
A Potrerillos & Sector Bajo & 81 & 250 & 3 & 0,3 \\
3.800 msm & Terraza Alta & 10 & 93 & 1 & 0,3 \\
Valles Las Taguas y Los Amarillos & Terraza Baja & 34 & 1.184 & 5 & 0,6 \\
3.700 msm & ARQ-15 & & 56 & 3 & 14 \\
\hline
\end{tabular}




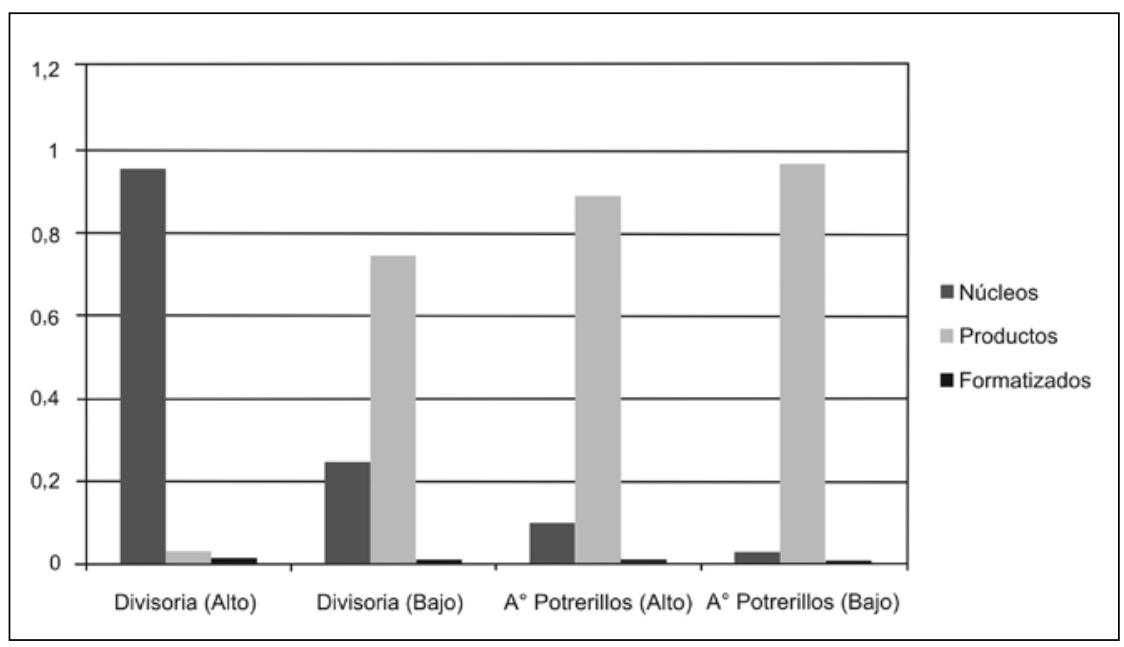

Figura 3. Composición de las muestras en sectores altos e intermedios. Composition of the samples at higher and intermediate sectors.

alta proporción de núcleos y formas naturales, en cambio en el sector bajo se destaca una presencia menor de núcleos y mayor de productos de talla. Esta tendencia se enfatiza en la terraza alta del arroyo Potrerillos; finalmente la terraza más baja, con vegetación y próxima al arroyo, muestra el mayor volumen de productos de talla y el más bajo en núcleos. Según se ha expresado, también la densidad de materiales en estos emplazamientos aumenta a medida que disminuye la altitud. Por otra parte la proporción de artefactos es muy baja en todos los sectores.

\section{Sectores bajos: los talleres líticos de los valles de Las Taguas y Los Amarillos}

En el área de los valles del río de Las Taguas y Los Amarillos (3.700 msm) se detectaron dos sitios arqueológicos con resguardos que se encuentran asociados a fuentes secundarias de rocas silíceas opacas blanquecinas y que tienen talleres en superficie: ARQ-15 y ARQ-26.

El sitio ARQ-15 se ubica en la margen derecha del río de Las Taguas, en una superficie pequeña e inclinada al pie de formaciones elevadas que cierran el valle hacia el este. Sobre la superficie de estas pendientes se observaron fragmentos naturales desprendidos con finas vetas de sílice blanquecina. Una de las posibles fuentes primarias para este sitio puede estar localizada en un sistema de vetas de composición silícea alojadas en la Formación Escabroso de edad Miocena media, que se encuentran muy próximas al sitio. La existencia de estas vetas ocurre principalmente en este sector del río de Las Taguas y se encuentra restringida a un radio de aproximadamente siete kilómetros tomando como centro el sitio ARQ-15. Estas vetas se desarrollan de manera posterior a los eventos volcánicos póstumos, de importancia en la región, debido a que se encuentran en relación de intrusión con las rocas de caja de distinta composición y edad. Poseen un desarrollo variado dependiendo de las rocas de caja en las cuales se alojan. Las más cercanas al sitio ARQ-15, consideradas como posible fuente primaria, son las de mejor desarrollo. Las mismas intruyen a la Formación Escabroso, la cual está compuesta principalmente por andesitas y brechas volcánicas de similar composición. Dichas vetas poseen un ancho máximo de $6 \mathrm{~m}$, mientras que su longitud alcanza la centena de metros (Figura 4).

Las vetas más conspicuas están compuestas por sílice cristalina de reemplazo de color blanco a gris claro. En gran cantidad de casos el propio proceso intrusivo genera un brechamiento de la roca de caja, dejando fragmentos de roca inmersos en la sílice. Su composición es variada pero en su mayoría corresponde a distintos tipos de sílice de relleno, con estructuras del tipo: diente de perro, microcristalinas y calcedónicas, entre otras. El segundo tipo de veta observado está conformado por un crecimiento gradual hacia el centro de la misma compuesto por sílice de reemplazo de color blanquecino, seguida por una sílice representada 


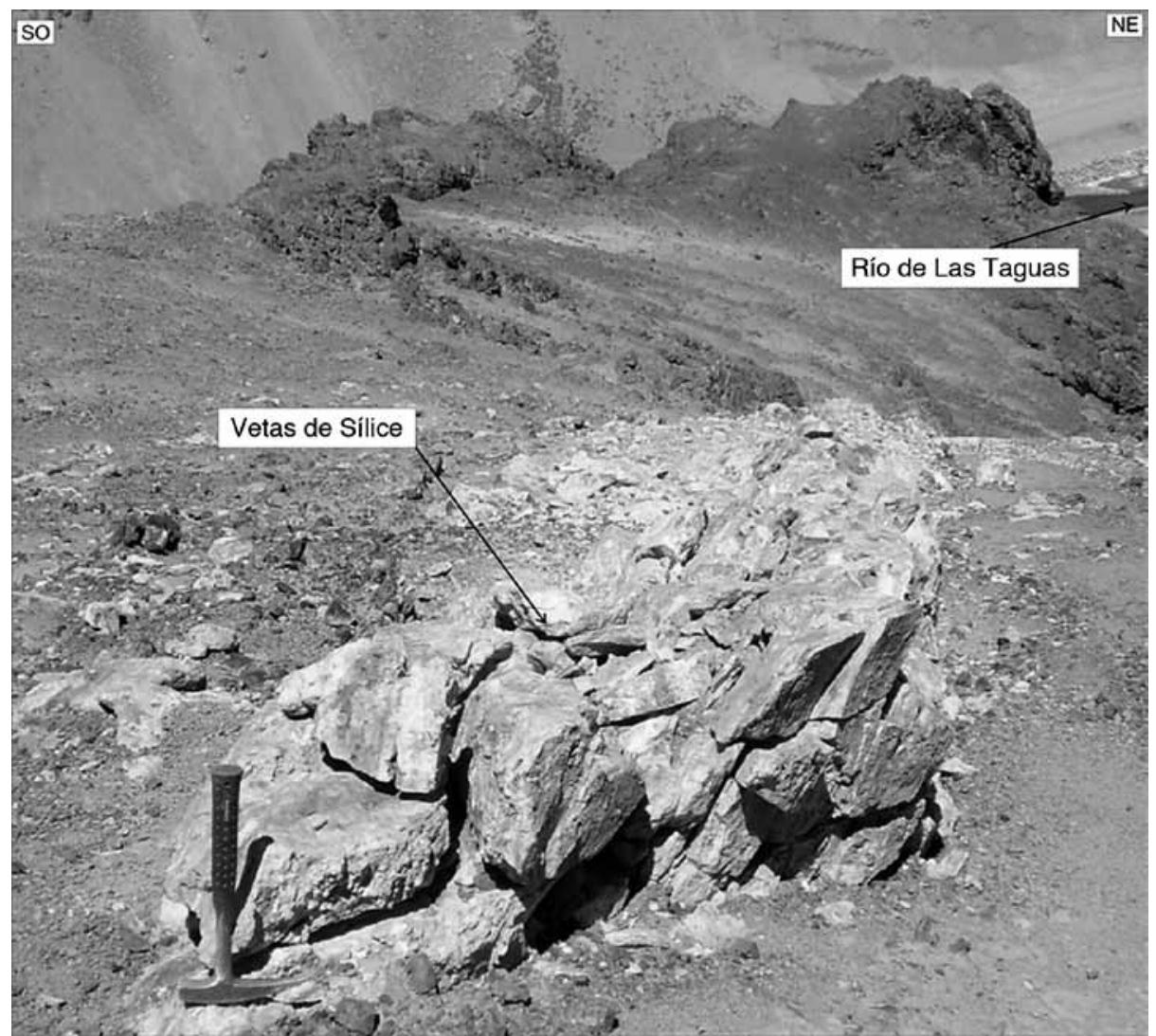

Figura 4. Afloramiento de una veta silícea próxima al río Las Taguas.

Outcrop of siliceous rock near the Las Taguas river.

por cristales de buen desarrollo (textura drúsica) culminando en el centro con una sílice calcedónica de color verdoso a gris claro. Un tercer tipo de veta observado en el lugar, aunque de menor desarrollo, está compuesto por cristales de sílice y adularia generando un bandeamiento característico. Los cristales de adularia (KAlSi3O8) poseen un color blanquecino y las agujas de sus cristales apuntan hacia el centro de la veta. Estas vetas corresponden a un sistema hidrotermal de baja sulfuración de probable edad Miocena Superior. Estas vetas, que constituyen una valiosa fuente primaria, se encuentran a una distancia menor a los $900 \mathrm{~m}$ en línea recta desde el sitio ARQ-15 y por corresponder a un sistema hidrotermal se encuentran restringidas en un radio de entre cinco y ocho kilómetros.

En el sitio ARQ-15 hay un pequeño acondicionamiento del espacio por medio de un pircado; se trata de un muro bajo de $1 \mathrm{~m}$ de largo que une dos grandes bloques naturales formando un reparo. La materia prima de la gran cantidad de productos líticos que pueden observarse en su superficie es precisamente esta silícea de color blanco a gris cremoso. Los materiales se dispersan en un área de 50 × 30 m. En el sitio se realizó una recolección en una unidad de $4 \mathrm{~m}^{2}$. Esto representa el 0,3\% de la superficie total del sitio. La muestra recuperada está integrada por 56 elementos líticos, lo que representa una densidad elevada de 14 elementos por $\mathrm{m}^{2}$ (Tabla 1).

El sitio ARQ-26 está ubicado en la margen derecha del arroyo Los Amarillos, en su curso alto. En un sector elevado hacia la pendiente sur del valle se encuentra un bloque errático de tres metros de alto, que genera resguardo de los vientos predominantes y en torno al cual se concentra una gran cantidad de material lítico (Figura 5). El sitio tiene un área de 30 x 15 m, se realizó una recolección en una unidad de $4 \mathrm{~m}^{2}$. Esto representa entonces un $0,9 \%$ del sitio y se recuperaron 426 piezas líticas, por tanto tiene una densidad muy elevada de 106,5 elementos por $\mathrm{m}^{2}$. La superficie del sitio presenta 


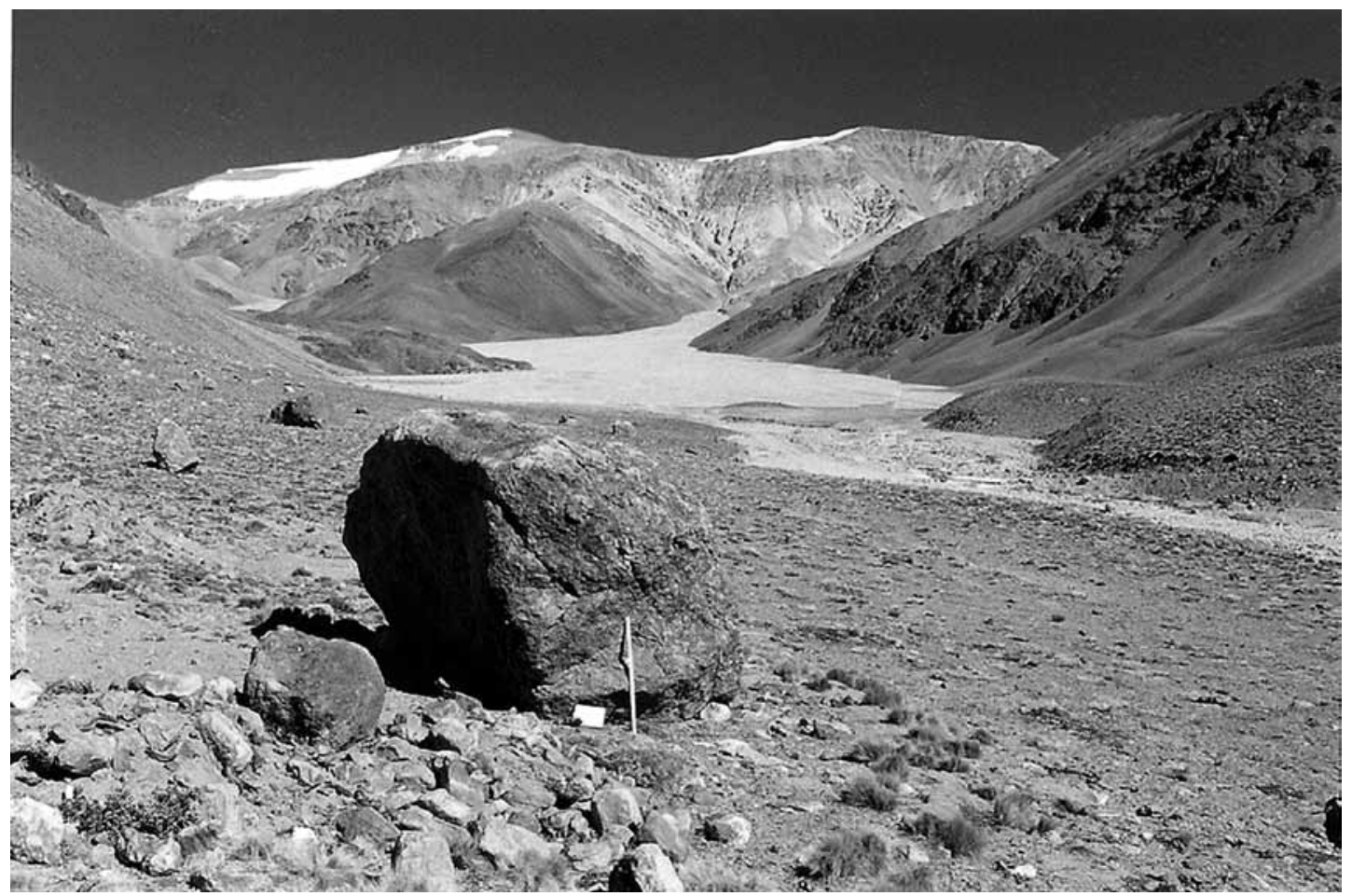

Figura 5. Sitio ARQ-26. Se observan en la imagen los flujos de detritos. ARQ-26 site. Figure show detritic flows.

un sedimento arenoso muy suelto, y además de realizar la recolección durante el año 2009, se dejó una señalización con estacas para controlar la unidad durante 2010. En la visita realizada en este año, se pudo observar que la superficie mostraba nuevamente una gran cantidad de productos de talla. Este proceso no está vinculado a reptaciones laterales, sino a procesos de desenterramiento generado por factores asociados a la remoción de sedimentos por acumulación de nieve y erosión eólica. Esto implica que la acumulación de productos en el sitio podría ser el resultado de sucesivas ocupaciones que alcanzan una cierta profundidad estratigráfica.

La materia prima predominante en los productos de talla del sitio es una silícea de color crema, cuya fuente primaria podría estar localizada en la cabecera del mismo valle glaciario en el cual se localiza el sitio. Las rocas que la componen son mayormente volcánicas habiendo participación de tobas riolíticas con gran cantidad de cristales de cuarzo, como así también existe la presencia de cuerpos graníticos que intruyen a estas secuencias. Como se puede observar en la Figura 5 existe un depósito de más de tres kilómetros de longitud, que es producto de sucesivos flujos de detritos que provienen de dicha cabecera. Estos flujos de detritos traen grandes bloques que son arrancados de los afloramientos en las cabeceras del valle y los transportan a lo largo del mismo. Esta situación genera que las fuentes se encuentren depositadas en las cercanías del sitio, sin tener que recorrer grandes distancias para llegar al afloramiento.

En la Figura 6 se comparan las secuencias de reducción lítica de los sitios con fuentes secundarias localizados en los sectores más bajos. Puede observarse en primer lugar que los productos iniciales de la talla, cómo núcleos o indiferenciados son muy bajos en los dos casos, esto marca una diferencia significativa con los sitios próximos a las fuentes primarias localizadas a mayor altitud. En el valle de Las Taguas se muestra una secuencia completa de reducción con el índice más elevado en lascas internas y la presencia de restos de microlascas, como producto de la regularización, e incluso algunos artefactos formatizados. En el caso del valle de Los Amarillos, es muy significativa la proporción de lascas externas, o con corteza, lascas internas y microlascas. La alta proporción de estos estadios, 
que implica la búsqueda de soportes, e incluso la regularización de artefactos y la ausencia total de los mismos, hacen que pueda considerarse como un área de taller asociado a esta fuente secundaria.

En estos emplazamientos más protegidos de los valles, con abundante presencia de recursos bióticos, disponibilidad de agua y espacios resguardados, se localizan entonces estos espacios específicos asociados a fuentes secundarias donde se encuentran las secuencias completas de reducción lítica y la mayor densidad de elementos arqueológicos en superficie (Figura 7). La abundancia de actividades de talla en los valles podría estar asociada tanto a la búsqueda y producción de soportes líticos, es decir a la existencia de talleres asociados a estas fuentes secundarias, como así también al uso recurrente a lo largo de varios milenios. Se verán a continuación algunas tendencias y datos preliminares que permiten proponer patrones temporales para la explotación de estos ambientes de altura.

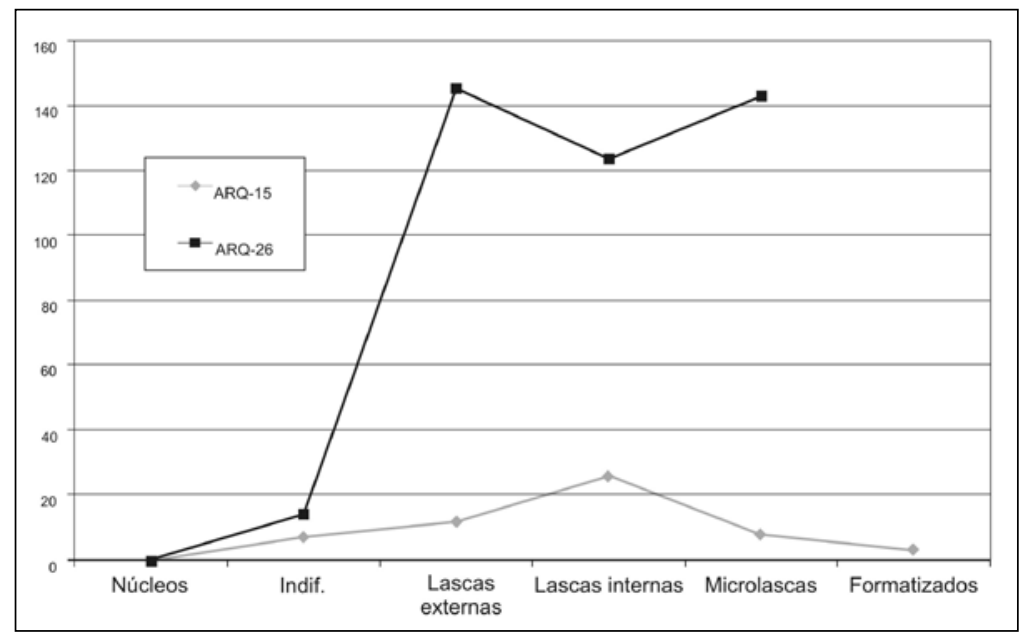

Figura 6. Secuencias de reducción lítica en los sitios ARQ-15 y ARQ-26.

Lithic reduction sequences in sites ARQ-15 and ARQ-26.

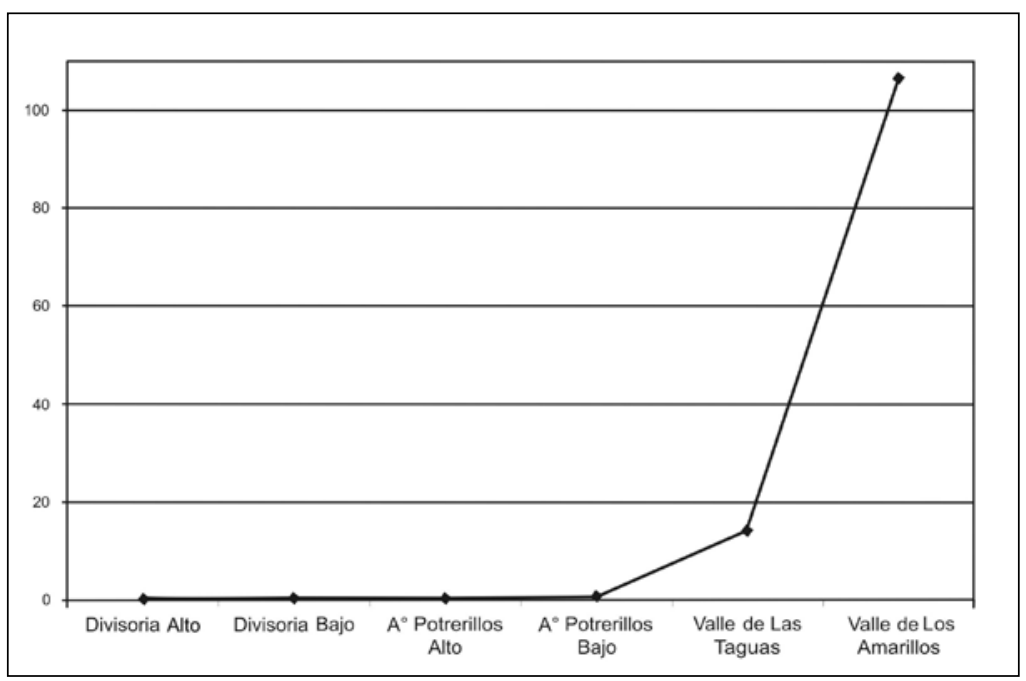

Figura 7. Densidad de elementos líticos según variación altitudinal.

Density of lithic elements according to altitudinal variation. 


\section{Aproximación a Tendencias Temporales en la Explotación del Área}

Para comenzar a indagar sobre patrones temporales en el uso humano de esta zona de la cordillera de los Andes, se realizará una caracterización general de la composición de las muestras líticas en el sitio que presenta la secuencia estratigráfica más profunda (ARQ-18). Se señalarán luego algunos datos paleoambientales importantes que permiten comprender desde cuándo y bajo qué condiciones climáticas este ambiente pudo ofrecer una alternativa para circuitos anuales que incluían la estación estival en cordillera.

La excavación de este sitio, para el que se han obtenido 18 dataciones radiocarbónicas, se realizó a partir de dos cuadrículas de $1 \mathrm{~m}^{2}$ y casi tres metros de profundidad hasta alcanzar la roca de caja de la formación del alero. Se han distinguido cinco componentes de ocupación delimitados por rasgos estratigráficos muy claros y fechados de techo y base (Tabla 2) (Cortegoso 2011). En cuanto al material lítico, la muestra está integrada por 16.364 elementos; de éstos, el 99\% son productos de talla. Hay escasa presencia de núcleos y artefactos formatizados. En la Figura 8 se grafica la distribución de los materiales líticos por extracciones, en la misma se han sombreado los componentes de ocupación.

Si bien el análisis de la totalidad de productos no se ha realizado aún, algunas propuestas preliminares se pueden establecer en función de la composición de la muestra por componentes y la caracterización de los artefactos formatizados recuperados en el sitio.

Comenzando por la ocupación más temprana (Componente V: ca. 8.900-8.000 años a.p.), se puede observar que representa un volumen muy bajo y discontinuo en el descarte de elementos líticos. En este componente se han observado entre los productos líticos algunas materias primas que son locales pero de calidades regulares, y también pequeños productos no locales. Teniendo en cuenta la excelente oferta de recursos en el área, podría considerarse que esta composición obedece a una etapa exploratoria del ambiente (sensu Franco 2004). Entre los escasos artefactos formatizados, destacamos la presencia de dos puntas de proyectil triangulares de tamaño pequeño (Figura 9). Esta tipología de proyectiles se ha registrado en ocupaciones tempranas de ambas vertientes cordilleranas, tanto en el norte y centro de Chile (Ampuero y Rivera 1971; Jackson et al. 2004; Núñez et al. 2002) como en regiones puneñas del noroeste argentino (Martínez 2007), y plantea un interesante desafío en cuanto a la procedencia de los primeros ocupantes de este ambiente de altura.

El componente IV (ca. 7.300-6.600 años a.p.) muestra el descarte más alto de toda la secuencia, tiene también presencia de núcleos y artefactos formatizados, en particular la continuidad de proyectiles triangulares pequeños (Figura 9). Dentro de los productos de talla se destaca una gran cantidad de lascas de adelgazamiento bifacial y abundante presencia de microlascas. Las materias primas

Tabla 2. Delimitación cronológica de los componentes de ARQ-18. Comparación con los períodos húmedos en cordillera estudiados por Veit (1996).

Chronological delimitation of the components of ARQ-18. Comparison with humid periods in cordillera defined by Veit (1996).

\begin{tabular}{ccc}
\hline Componentes ARQ-18 & Fechados radiocarbónicos años a.p. & $\begin{array}{c}\text { Períodos húmedos en Cordillera años a.p. } \\
\text { (Veit 1996) }\end{array}$ \\
\hline Componente I & $1.540 \pm 60$ (LP-2085) carbón & $3.000-1.800$ \\
& $2.980 \pm 70$ (LP-1748) carbón & \\
Componente II & $3.720 \pm 60$ (LP-1847) carbón \\
Componente III & $4.310 \pm 90$ (LP- 1973) carbón \\
& $5.120 \pm 60$ (LP-1840) carbón \\
Componente IV & $6.690 \pm 90$ (LP- 1976) carbón & \\
& $7.300 \pm 90$ (LP-1857) carbón & 7.300 \\
Componente V & $8.070 \pm 140$ (LP-1977) carbón \\
& $8.950 \pm 100$ (LP-1815) carbón & \\
\hline
\end{tabular}




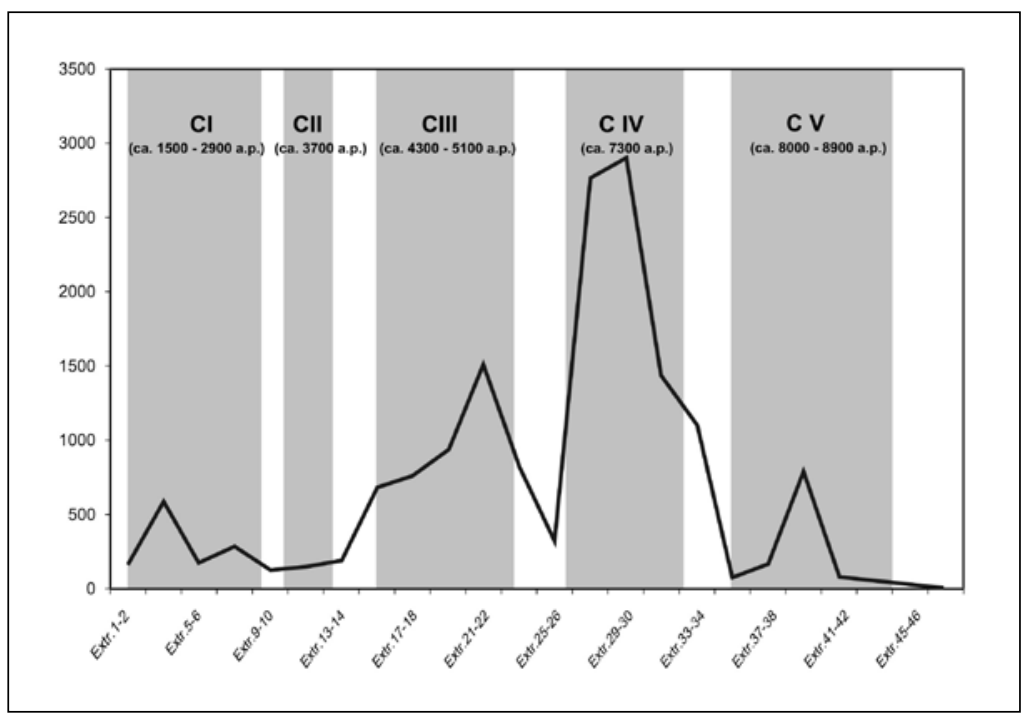

Figura 8. Distribución de elementos líticos por extracción en ARQ-18. Se sombrean los cinco componentes registrados en el sitio.

Distribution of lithic elements per level in ARQ-18. Shading shows the five components registered on the site.

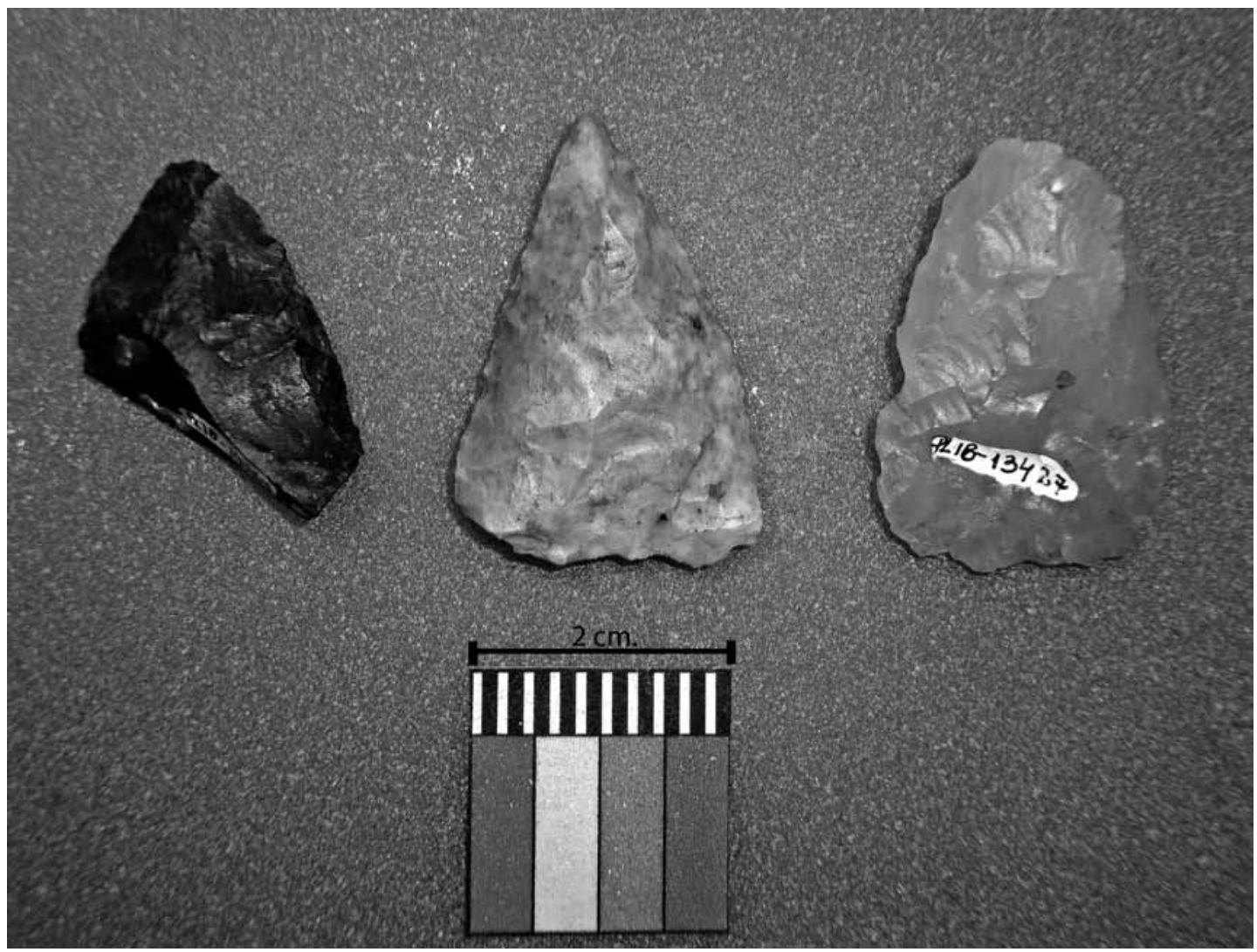

Figura 9. Puntas de proyectil triangulares registradas en los componentes más tempranos (ver Tabla 2). Izquierda y centro corresponden al componente V; derecha componente IV.

Triangular projectile points recorded in the earliest components (see Table 2). Left and center: component V; right: component IV. 
más abundantes en este conjunto son las sílices de colores crema de excelente calidad cuyas fuentes secundarias se encuentran, como se ha analizado, en el propio valle de Las Taguas. El registro sería equiparable entonces a una etapa de colonización del área (sensu Franco 2004) que se manifiesta en un conocimiento de las fuentes del área y una producción local de artefactos.

En el componente III (ca. 5.100-4.300 años a.p.) hay mayor variabilidad de artefactos, aun cuando se registra una disminución en las actividades de talla dentro del sitio. La gran diversidad de materias primas representadas en los artefactos podría señalar una estrategia de uso recurrente de este espacio. El sitio muestra en esta ocupación un acondicionamiento realizado a través de un gran pozo rodeado de grandes rocas que delimita un espacio interno con gran presencia de carbón. Entre los artefactos se destaca la presencia de puntas triangulares grandes, que también están registradas para esta cronología en sitios de San Juan (Gambier 1985) y de Chile (Méndez et al. 2009). También en este componente se han detectado ciertos indicadores arqueofaunísticos que permiten proponer que el área habría comenzado a ser explotada por pastores (Gasco 2009).

El componente II (ca. 3.700 años a.p.) señala una caída abrupta en el uso del sitio, el registro material es muy exiguo en todas sus categorías y hay muy pocos restos de talla en el sitio. Se registró la presencia de muy pocos artefactos formatizados, algunos realizados sobre materias primas que no son locales.

El componente más tardío (Componente I: ca. 2.900-1.500 años a.p.) mostraba un acondicionamiento del sitio similar al del componente III, y aun cuando manifiesta una presencia más baja de productos de talla, tiene un conjunto artefactual similar al recuperado en dicho componente. Entre los cabezales líticos el tipo recurrente son puntas triangulares de distinto tamaño. Las similitudes más destacables que podrían indagarse en este caso serían lo que se ha denominado para la vertiente chilena como: Tradición Interior de Puntas Triangulares (Méndez y Jackson 2008).

Algunos datos paleoambientales estudiados para regiones próximas muestran que el uso humano de estos ambientes de altura ha estado condicionado por dos variables importantes: la apertura del área luego de la retirada de los hielos, y la disponibilidad de agua en cordillera que se correlaciona con ciclos áridos en los sectores bajos de Chile.

De acuerdo con estudios realizados en el Valle del Encierro, en un área muy próxima de la vertiente occidental, esta latitud andina habría sido afectada por avances de glaciares hasta 9.300 años a.p. (Zech et al. 2006). Esto permitiría explicar que la ocupación registrada más antigua tenga casi 9.000 años.

Desde que comenzó a ocuparse este espacio, es destacable la correlación que existe con los factores ambientales que marcan fluctuaciones de aridez. En la región el período más árido del Holoceno está registrado entre 7.500 y 6.000 años a.p. (Maldonado y Rozas 2008; Veit 1996). La aridez extrema de Holoceno medio en el área andina es un tema que se ha abordado a escala regional en los últimos años y que ha mostrado hiatos o vacíos arqueológicos en vastas regiones (Zárate et al. 2005). En este contexto, cobran particular relevancia los períodos con mayor humedad en Cordillera que Veit (1996) ha establecido para el Holoceno específicamente en el Norte Chico chileno: el primero alcanza hasta los 7.300 años a.p., el segundo 5.000-3.700 y el tercero 3.000-1.800. En cuanto a este último período otros estudios también han señalado que la humedad efectiva en el extremo sur de los Andes Centrales $\left(29^{\circ} \mathrm{S}\right)$ habría sido entre un 20 y un $30 \%$ superior a las actuales condiciones (Grosjean et al. 1997).

Según estos estudios, la disponibilidad de agua en cordillera en estos períodos, consecuencia de los vientos del Pacífico, justamente se correlaciona con extremos áridos en los sectores más bajos, por tanto la ocupación estival de estos ambientes permitiría afrontar el riesgo de la estación más seca en la vertiente occidental cordillerana.

Los períodos y los fechados obtenidos en estos estudios paleoambientales, particularmente los de Veit (1996), muestran una correlación llamativa con el registro humano del valle de Las Taguas (Tabla 2). Según estos datos, cuando la cordillera tuvo disponibilidad hídrica y los valles chilenos afrontaron períodos muy secos, en el valle del río de Las Taguas se generaron los registros más marcados de presencia humana.

\section{Discusión y Perspectivas}

La región limítrofe argentino-chilena y los valles orientales en esta latitud presentan una excelente 
disponibilidad de materias primas; en especial una amplia variedad de rocas silíceas criptocristalinas. La explotación de estos recursos locales representa casi la totalidad de las materias primas presentes en los sitios que se están estudiando en la región. En cuanto a los patrones espaciales que han podido analizarse, el registro de superficie muestra una variabilidad en densidad y composición que está relacionada tanto con la calidad de las materias primas en el área, como con la altitud y las condiciones de los ambientes. En la divisoria de vertientes se localizaron fuentes primarias y se registraron fragmentos naturales y núcleos. Este ambiente es el menos adecuado para la instalación humana, está por encima de la cobertura vegetal y se caracteriza por condiciones ambientales extremas, especialmente térmicas, que afectan tanto la ocupación humana como la calidad de las materias primas. Es el área con más baja densidad de materiales, en los cuales resulta difícil diferenciar procesos de fracturación naturales y antrópicos.

Los sectores intermedios muestran mayor densidad, en especial en la terraza más baja del arroyo Potrerillos. Aparece allí una gran diversidad de rocas, provenientes de fuentes primarias y secundarias de excelente calidad para la talla. Finalmente en los sitios del valle se encuentran sólo fuentes secundarias y talleres de producción lítica con una densidad muy grande que podría ser resultado de procesos diacrónicos de formación. Las características del registro de los valles son importantes para analizar la recurrencia en la explotación de estos ambientes a lo largo de varios milenios.

Las tendencias temporales en el uso de estos ambientes cordilleranos han comenzado a proponerse en función del análisis preliminar de la composición del registro lítico proveniente de un sitio con una secuencia de ca. 9.000 años radiocarbónicos. El área habría comenzado a explorarse inmediatamente después de la retirada de los hielos del último avance del límite Pleistoceno-Holoceno, según está registrado en el Valle del Encierro. Las ocupaciones humanas a lo largo del Holoceno muestran una continuidad en el uso del área y sus recursos líticos, como así también variabilidad en las estrategias de producción. A lo largo de la secuencia se produjeron cambios importantes en la subsistencia de quienes explotaron estacionalmente estos ambientes; algunos indicadores de la presencia temprana de pastores están evaluándose en este registro. Los conjuntos líticos muestran frecuencias variables en los últimos milenios y también la continuidad en las prácticas de caza, según lo manifiesta la presencia de puntas de proyectil a lo largo de toda la secuencia. $\mathrm{La}$ correlación del uso del área con períodos húmedos en cordillera y secos en la vertiente occidental, junto con la mejor accesibilidad desde esa zona y las características preliminares observadas en el registro lítico, permiten proponer que el área habría sido parte de circuitos de movilidad que incluían dicha vertiente.

La profundización de los estudios en la región permitirá explicar las diferencias en la explotación cordillerana entre las tempranas ocupaciones de cazadores recolectores y las sociedades productoras de alimentos más tardías. También es esperable que futuros análisis permitan precisar patrones temporales en el uso de estos ambientes de altura más complejos y variables a lo largo del Holoceno. La región pudo haberse explotado también desde las regiones puneñas del norte argentino, como desde los valles más bajos de la provincia de San Juan; incluso podrían plantearse períodos con vinculación o contacto entre comunidades instaladas de forma permanente a uno y otro lado de la cordillera, como se ha registrado para períodos tardíos. Estos temas podrán indagarse en los próximos años a partir de la rica base material recuperada en esta zona limítrofe para la que no se registraban antecedentes.

Agradecimientos: A los organizadores del Simposio "Fuentes de materias primas líticas: estrategias de aprovisionamiento, uso y circulación", realizado en el XVIII Congreso Nacional de Arqueología Chilena. Los trabajos de campo fueron financiados gracias a un convenio entre la empresa BEASA y CONICET. Las investigaciones cuentan con el apoyo y financiamiento de: CONICET (PIP CONICET 114-200801-00177), ANPCyT (PICT2006-N ${ }^{\circ} 421$ ), Secyt-UNCuyo (Proyecto 06/G502). A los revisores externos anónimos que evaluaron el manuscrito. 


\section{Referencias Citadas}

Ampuero, G. y M. Rivera 1971. Secuencia arqueológica del alero rocoso de San Pedro Viejo de Pichasca. Boletín del Museo Arqueológico de La Serena 14:45-69.

Andrefsky, W. 2009. The Analysis of Stone Tool Procurement, Production, and Maintenance. Journal of Archaeological Research 17:65-103.

Aschero, C. 1983. Ensayo para una clasificación morfológica de artefactos líticos. Informe a CONICET. Manuscrito en posesión del los autores.

Cortegoso, V. 2008. Disponibilidad de recursos líticos en el noroeste de Mendoza: cambios en la organización tecnológica en la cuenca del río Blanco. Cazadores y Recolectores del Cono Sur. 3:95-113.

- - - 2011. Las Taguas Arq. 18. Estratigrafía, secuencias temporales y ocupaciones humanas. En Arqueología del Límite Cordillerano. Proyecto Lama, San Juan, editado por V. Durán y V. Cortegoso. Universidad Nacional de Cuyo, Mendoza, en prensa.

Franco, N. 2004. La organización tecnológica y el uso de escalas espaciales amplias. El caso del Sur y Oeste de Lago Argentino. En Temas de Arqueología, Análisis Lítico, compilado por A. Acosta, D. Loponte y M. Ramos, pp. 101-144. Universidad Nacional de Luján, Luján.

Gambier, M. 1985. La Cultura de Los Morrillos. Instituto de Investigaciones Arqueológicas y Museo. Universidad Nacional de San Juan, San Juan.

Gasco, A. 2009. Variabilidad osteométrica de camélidos a lo largo del Holoceno en Las Taguas-ARQ18 (San Juan-Argentina). Ponencia presentada en 13 Congreso de Antropología Colombia. 1er Encuentro Latinoamericano de Zooarqueología. Bogotá.

Grosjean, M., B. Valero Garcés, M. Geyh, B. Messerli, U. Schotterer, H. Schreier y K. Kelts 1997. Mid-and late-Holocene limnogeology of Laguna del Negro Francisco, Northern Chile, and its palaeoclimatic implications. The Holocene 7:151-159.

Jackson, D., C. Méndez y P. De Souza 2004. Poblamiento Paleoindio en el norte-centro de Chile: Evidencias, problemas y perspectivas de estudio. Complutum 15:165-176.

Kuhn, S.L. 2004. Upper Paleolithic raw material encomies at Ucagizli cave. Turkey. Journal of Anthropological Archaeology 23:431-448.
Maldonado, A. y E. Rozas 2008. Clima y paleoambientes durante el Cuaternario Tardío en la Región de Atacama. En Libro Rojo de la Flora Nativa y de los Sitios Prioritarios para su Conservación. Región de Atacama, editado por F. Squeo, F. Arancio y J. Gutiérrez, pp. 293-304. Ediciones Universidad de La Serena, La Serena.

Martínez, J. 2007. Ocupaciones humanas tempranas y tecnología de caza en Antofagasta de la Sierra, Puna Meridional Argentina (10000-7000 AP). Cazadores y Recolectores del Cono Sur 2:129-150.

Méndez, C. y D. Jackson 2008. La ocupación prehispánica de Combarbalá (Norte Semiárido, Chile): una propuesta sintética. Chungara Revista de Antropología Chilena 40:107-119.

Méndez, C., A. Troncoso, D. Jackson y D. Pavlovic 2009. Movilidad y uso del espacio entre cazadores recolectores tardíos en espacios cordilleranos del Norte Semiárido de Chile. Intersecciones en Antropología 10:311-326.

Nelson, M. 1991. The study of technological organization. En Archaeological Method and Theory, editado por M. Schiffer, vol. 3, pp. 57-100. University of Arizona Press, Arizona.

Núñez, L., M. Grosjean e I. Cartajena 2002. Human occupations and climate change in the Puna de Atacama, Chile. Science 298:821-824.

Steffen, A., E. Skinner y P. Ainsworth 1998. A view to the core technological units and debitage analysis. En New Issues in Archaeology. Measuring Time, Space and Material, editado por A.F. Ramenofsky y A. Steffen, pp .131-146. University of Utah Press.

Veit, H. 1996. Southern Westerlies during the Holocene deduced from geomorphological and pedological studies in the Norte Chico, Northern Chile $\left(27-33^{\circ}\right.$ S). Palaeogeography, Palaeoclimatology, Palaeoecology 123:107 119.

Zárate, M., G. Neme y A. Gil 2005. Mid Holocene paleoenvironments and human occupation in Southern South America. Quaternary International 132:1-3.

Zech, R., C. Kull y H. Veit 2006. Late Quaternary glacial history in the Encierro Valley, northern Chile $\left(29^{\circ} \mathrm{S}\right)$, deduced from $10 \mathrm{Be}$ surface exposure dating. Palaeogeography, Palaeoclimatology, Palaeoecology 234:277-286. 